\title{
SISTEMATIZACIÓN, DOCUMENTACIÓN Y DIVULGACIÓN VIRTUAL DE LOS JUEGOS RECREATIVOS TRADICIONALES DE LA CALLE DEL MUNICIPIO DE CALDAS - ANTIOQUIA 1981-2014
}

Systematization, documentation and virtual dissemination of Traditional Recreational Games Street in the Caldas municipality- Antioquia 1981-2014

Sistematização, documentação e divulgação virtual das brincadeiras Tradicionais de rua do municipio de Caldas - Antioquia 1981-2014
Santiago Santa Ríos (1)
William Moreno Gómez (2)
Álvaro Alexander Mejía Henao (3)
(1) Universidad de Antioquia, Colombia. Fono: +573122255914. Correo electrónico: ssan77a@ gmail.com.
(2) Universidad de Antioquia, Colombia. Fono: +573122586741. Correo electrónico: williammorenoiuef@gmail.com
(3) Universidad de Antioquia, Colombia. Fono +573103522511. Correo electrónico: aamhen@ hotmail.com

\section{RESUMEN}

Este texto da cuenta de dos asuntos: (1) una sistematización de la base fotográfica y video gráfica levantada en el trabajo de campo de la investigación que sobre los Juegos recreativos tradicionales de la calle viene realizando el PES en el municipio de Caldas desde el año $2009^{2}$; ésta sistematización establece una clasificación, una categorización y una significación básica de dichos documentos, y (2) la construcción de una Webquest (herramienta virtual) para uso educativo- comunicacional, aprovechando la sistematización de los Juegos Recreativos Tradicionales de la Calle (JRTC) mencionada anteriormente. Esta sistematización y la Webquest configuran una estrategia que permite la inserción del semillero PES en el proceso de recuperar y divulgar el patrimonio histórico y cultural de los JRTC (1981-2014). El proyecto en esta fase constituye un sistema de información virtual comprometido en el proyecto CODI "Juegos de la calle una buena práctica referenciable en los procesos de educación del cuerpo en Colombia”

\footnotetext{
${ }^{1}$ Resultados parciales de este proyecto han sido presentados en el Evento académico de los Juegos Tradicionales de la Calle, en sesión ampliada del Consejo del Municipio de Caldas, en sesión ordinaria del comité organizador de los JRTC, en Seminario académico JRTC en la Universidad de la Salle, evento académico JRTC IE Ciro Mendía, en Encuentro nacional de semilleros de investigación en la Universidad de Antioquia y en la Cátedra inaugural del Instituto Universitario de Educación Física de la Universidad de Antioquia sobre los JRTC.

2 Estudio adscrito a macro proyecto de investigación denominado "Documentación, sistematización e interpretación de buenas prácticas pedagógicas en los procesos de educación del cuerpo en la escuela" con participación de la Universidad de Antioquia (Col.), Universidades federales de Santa Catarina, Río de Janeiro, Paraná y la Universidad Nacional de La Plata (Arg.) ejecutado con el apoyo financiero de la Universidad de Antioquia, proyecto CODI 2012-2014 e INDEPORTES Antioquia. Investigadores: William Moreno, Sandra Pulido, Néstor Díaz, Guillermo Betancur, Álvaro Mejía y Santiago Santa.
} 
Palabras clave: sistematización, juego tradicional.

\section{SUMMARY}

This text is about two topics: (1) a systematization of the photographic and videographic base built in the fieldwork research on traditional recreational street games done by the PES in the municipality of Caldas since 2009; this systematization establishes a classification , categorization and basic significance of these documents, and (2) building a Webquest (virtual tool) to educational-communicational use, taking advantage of the systematization of Traditional Recreational Street Games ( TRSG ) mentioned above. This systematization and Webquest form a strategy that allows insertion of the PES in the process of recovering and disseminating the historical and cultural heritage of the TRSG (1981-2014). The project at this stage is a virtual information system involved in the project CODI. "Street Games, a good referable practice in the education processes of the body in Colombia"

Keywords: Systematization, traditional game.

\section{RESUMO}

Este texto da conta de dois questões: (1) uma sistematização da base fotográfica e vídeo gráfica levantada no trabalho de campo da pesquisa que sobre as brincadeiras tradicionais de rua vem fazendo o PES no município de Caldas desde o ano 2009³; esta sistematização estabelece uma classificação, uma categorização e uma significação básica de ditos documentos, e (2) a construção de uma Webquest (ferramenta virtual) para uso educativocomunicacional, aproveitando a sistematização das brincadeiras tradicionais de rua mencionada anteriormente. Esta sistematização e a Webquest configuram uma estratégia que permite a inserção do grupo PES no processo de recuperar e divulgar o patrimônio histórico e cultural das brincadeiras tradicionais de rua (1981-2014). O projeto nesta etapa constitui um sistema de informação virtual comprometido no projeto CODI "Brincadeiras de rua uma boa prática referenciável nos processos de educação do corpo na Colômbia”

Palavras-chave: Sistematização, brincadeira tradicional.

\footnotetext{
${ }^{3}$ Estudo vinculado ao macroprojeto de pesquisa "Documentação, sistematização e interpretação de boas práticas pedagógicas nos processos de educação dos corpos na escola” com participação da Universidade de Antioquia (Col.), Universidades federais de Santa Catarina, Rio de Janeiro, Paraná e a Universidade Nacional de La Plata (Arg.) feito com o apoio financeiro da Universidade de Antoquia, projeto CODI 2012-2014 e INDEPORTES Antioquia. Pesquisadores: William Moreno, Sandra Pulido, Néstor Díaz, Guillermo Betancur, Álvaro Mejía y Santiago Santa
} 


\section{INTRODUCCIÓN}

Los Juegos Recreativos Tradicionales de la calle se celebran ininterrumpidamente en el Municipio de Caldas (Antioquia) desde hace treinta y tres años. Hoy, cuando se cumplen algo más de tres décadas de existencia, el grupo de investigación Prácticas Corporales: Sociedad, Educación-Currículo (PES) de La Universidad de Antioquia, interesado por el patrimonio histórico del campo de la educación física, el deporte y la recreación, a través de un proyecto de investigación internacional apoyado por el CNPq (Br.), INDEPORTES, Universidad de Antioquia, el gobierno municipal y la Mesa de Educación Física de Caldas, ha identificado que: (1) Los JRTC a pesar de ser una actividad de masas y de alto impacto educativo, se reduce hoy a una experiencia de alcance local que puede proyectarse a nivel nacional e internacional; (2) Hay un gran cumulo de información sobre JRTC diseminada en las oficinas de gobierno, bibliotecas y archivos personales; (3) La información producida a lo largo de los JRTC está consignada en un formato tradicional que desconoce el potencial de las TICs; (4) Existe una base informativa de JRTC que permite aprovechar el potencial educativo,turístico y promocional de esta práctica.

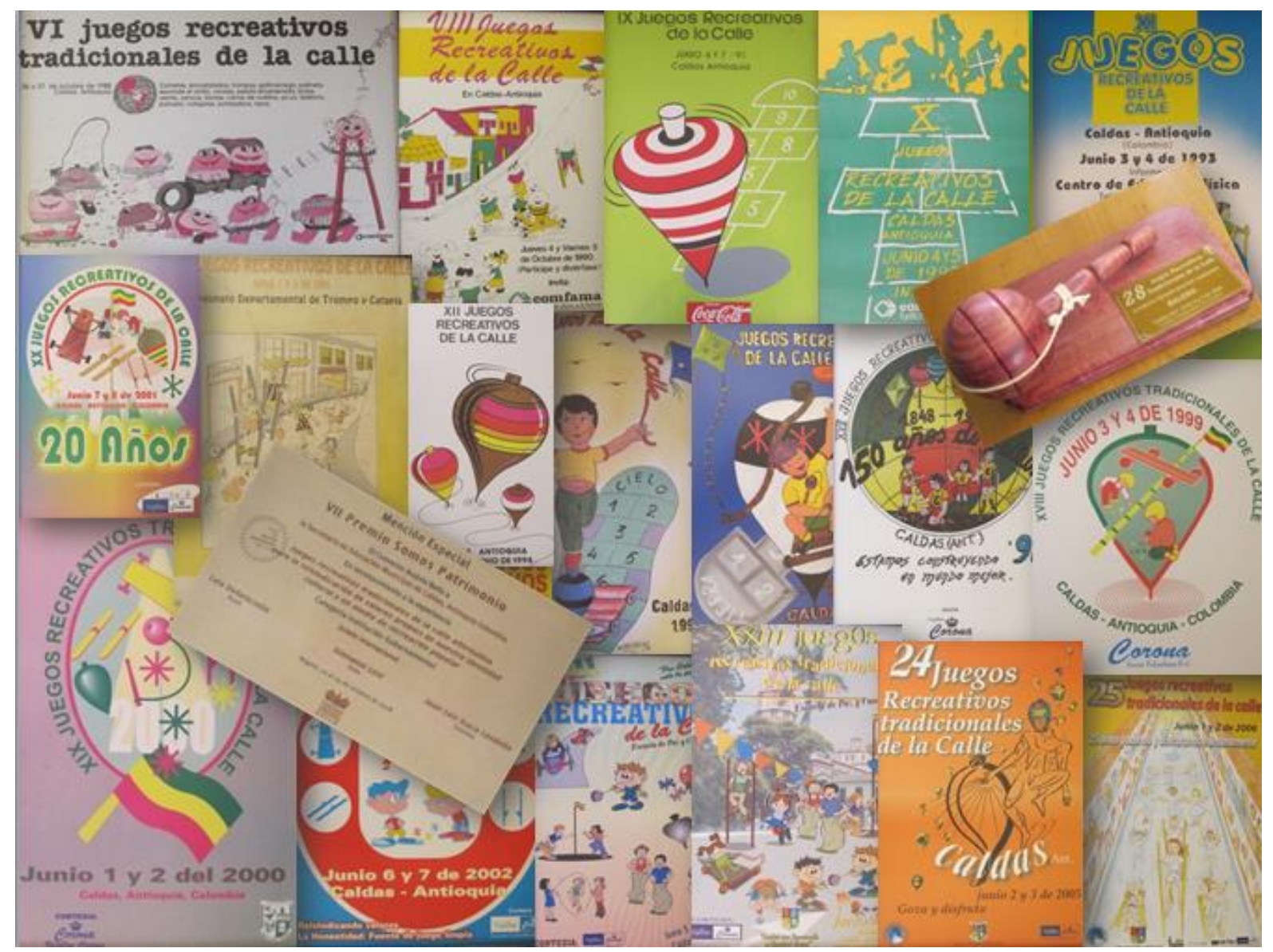

Fotografía 1. Treinta y tres años jugando... 
Los JRTC consisten en una experiencia recreativa cultural que se desarrolla todo el año y que se escenifican en Caldas la primera semana de Junio, contando con la participación de las instituciones educativas de Caldas, del departamento, del país y con alguna representación internacional. Muestran vigencia y continuidad histórica, han ganado el estatuto de política pública municipal en Caldas y van encaminados a convertirse en política pública en Antioquia y Colombia; por ello se constituye en una práctica significativa y emblemática que quiere ser promocionada como una "Buena Práctica" de alto valor educativo que sirve como: (1) Medio para la formación ciudadana y para el desarrollo de valores asociados a la convivencia y la salud; (2) Aliciente para la creación, favorecimiento y sostenibilidad de otras prácticas similares; (3) Referente para actividades con fines académicos en colegios y universidades (Contenido curricular). Este proyecto posee dos componentes, (1) Sistematización y documentación de la base fotográfica y video gráfica de los JRTC, y (2) Webquest de JRTC 1981-2014.

\section{PLANTEAMIENTO DEL PROBLEMA Y JUSTIFICACIÓN}

El objeto de conocimiento de este estudio es la sistematización y la divulgación efectiva de la experiencia JRTC del municipio de Caldas en el Departamento de Antioquia (Colombia).

Este objeto define el proceso de sistematización de la información JRTC, así como la reconfiguración de una herramienta que permita comunicar una experiencia educativa y potente, que sin la divulgación se reduce a una práctica desaprovechada para los propósitos sociales, culturales y formativos más elevados de los caldeños. El proceso investigativo consta de dos momentos: el primero se corresponde con el proceso de organización de la documentación de los Juegos desde el año 1981 hasta 2014, el segundo, tiene que ver con la exhibición virtual de dicha información para que los caldeños se proyecten hacia el exterior y para que la comunidad externa acceda fácilmente a la cultura caldeña.

Los JRTC constituyen una experiencia recreativa cultural que se desarrolla todo el año y que se escenifica en Caldas como certamen recreo deportivo desde 1981, pero carecen de una recopilación y sistematización de la información producida a lo largo de su existencia; igualmente, carece de una herramienta virtual que favorezca su divulgación. Producto y acción divulgativa que permitan su arraigo, su sostenibilidad, su proyección socio cultural y sobre todo su uso educativo. 


\begin{tabular}{|c|c|}
\hline SITUACIÓN & ACCIÓN (Evaluación) \\
\hline $\begin{array}{l}\text { JRTC posee treinta y tres años de existencia } \\
\text { ininterrumpida. }\end{array}$ & $\begin{array}{l}\text { Sistematizar información previa JRTC (Registro en } \\
\text { base de datos/ficha documental). }\end{array}$ \\
\hline $\begin{array}{l}\text { Se cuenta con una experiencia recreativa con algo } \\
\text { más de tres décadas de existencia, con } \\
\text { reconocimiento prevalente de la comunidad local, } \\
\text { escasamente a nivel nacional e internacional. }\end{array}$ & $\begin{array}{l}\text { Dar a conocer JRTC a nivel nacional e internacional } \\
\text { (Número de visitas a la Webquest activa, Webquest } \\
\text { enlazada en bases nacionales e internacionales en } \\
\text { 2014). }\end{array}$ \\
\hline $\begin{array}{l}\text { Organización y clasificación de información que } \\
\text { posibilite el registro en plataforma Excel y en la } \\
\text { Webquest JRTC. }\end{array}$ & $\begin{array}{l}\text { Sistematización de la base fotográfica y video gráfica } \\
\text { de los JRTC (Alojada en la Webquest). }\end{array}$ \\
\hline $\begin{array}{l}\text { Se cuenta con una información de JRTC } \\
\text { proveniente de investigación previa. }\end{array}$ & $\begin{array}{l}\text { Ingresar información a plataforma Webquest JRTC } \\
\text { (Documentación en base Excel y fijada en Webquest } \\
\text { JRTC.). }\end{array}$ \\
\hline Webquest JRTC. & $\begin{array}{l}\text { Ingresar información documental (escritural, } \\
\text { fotográfica y video gráfica) a Webquest (Link activo } \\
\text { de la Webquest JRTC: www.juegosdelacalle.com). }\end{array}$ \\
\hline $\begin{array}{l}\text { Herramienta virtual JRTC en formato Webquest, } \\
\text { para uso educativo y comunicacional. }\end{array}$ & $\begin{array}{l}\text { Diseño de Webquest (Link Webquest JRTC: } \\
\text { www.juegosdelacalle.com). }\end{array}$ \\
\hline $\begin{array}{l}\text { Se cuenta con producto investigativo sobre JRTC } \\
\text { (Tesis de Especialización y Maestría, libros, } \\
\text { capítulos de libro, artículos de revista). }\end{array}$ & $\begin{array}{l}\text { Ingresar productos a plataforma Webquest JRTC } \\
\text { (Número de visitas a los productos). }\end{array}$ \\
\hline $\begin{array}{l}\text { Hay compromiso de la Alcaldía de Caldas de } \\
\text { colocar una maquina servidor para el proceso de } \\
\text { divulgación JRTC }\end{array}$ & $\begin{array}{l}\text { Instalar maquina servidor en la Biblioteca municipal } \\
\text { de Caldas }\end{array}$ \\
\hline
\end{tabular}

Tabla 1. Entre la sistematización y la divulgación

Entre las causas directas del problema podemos encontrar: La existencia de grandes volúmenes de información escrita, fotográfica y video gráfica almacenada sin ningún criterio y con utilización muy reducida, lo que produce la invisibilización social y cultural de los JRTC. Pese a ello, estas justas han demostrado su valor social y educativo; constituyendo una "Buena Práctica" que podría ser referente de otros desarrollos educativos en el departamento y en el país. Hay otras causas relacionadas con el asunto investigativo: El desaprovechamiento de la documentación escrita y gráfica para la formación de maestros y para la educación básica y universitaria; el desconocimiento de la experiencia ludo deportiva propia y de su potencial educativo-curricular; subvaloración de lo propio y mitificación de lo "extraño".

Esta situación puede estar generando: una pérdida de los JRTC como política pública que intenta afirmarse en el nivel municipal y departamental, generándose así un bajo impacto 
social de una inversión pública que convoca a múltiples oficinas del estado tales como COLDEPORTES, Secretaría de Educación Departamental y Municipal, INDEC, INDEPORTES (...); un desconocimiento de los mismos caldeños acerca del potencial educativo, turístico y recreativo que contienen los JRTC, y posiblemente, una reducción de los JRTC a una condición de "espectáculo local" pleno en activismo, pero pobre en la promoción de educación ciudadana.

La concreción de este proyecto permite a la comunidad local y externa, tener una base documental de Juegos. El primer componente (sistematización y documentación), consiste en una base Excel que contiene la información textual (escritural, fotográfica y video gráfica) recogida en las investigaciones PES (2009-2011 y 2012-2014) ${ }^{4}$ y donaciones de fotografía con registro profesional. El segundo componente consiste en una Webquest, hoja de trabajo URL con propiedades Interactivas, informativas, educativas, de recepción, enlace y gestión; que facilitan el uso educativo comunicacional de los JRTC.

Esta problematización nos lleva a un interrogante orientador ¿Cuáles son los procesos y herramientas necesarias para sistematizar y divulgar los JRTC del Municipio de Caldas

\section{- Antioquia 1981-2014?}

La pregunta guía el proceso de sistematización (organización y visibilización del patrimonio histórico JRTC) y de configuración de un medio educativo pertinente para la estimulación de la memoria histórico cultural; sistematización y configuración de herramienta virtual claves para la divulgación y promoción de los JRTC en el contexto local, regional, nacional e internacional.

\footnotetext{
${ }^{4}$ Este sub proyecto está adscrito a la investigación PES "Documentación, sistematización e interpretación de buenas prácticas pedagógicas en los procesos de educación del cuerpo en la escuela" (2012-2014).
} 
3. Estado de la cuestión.

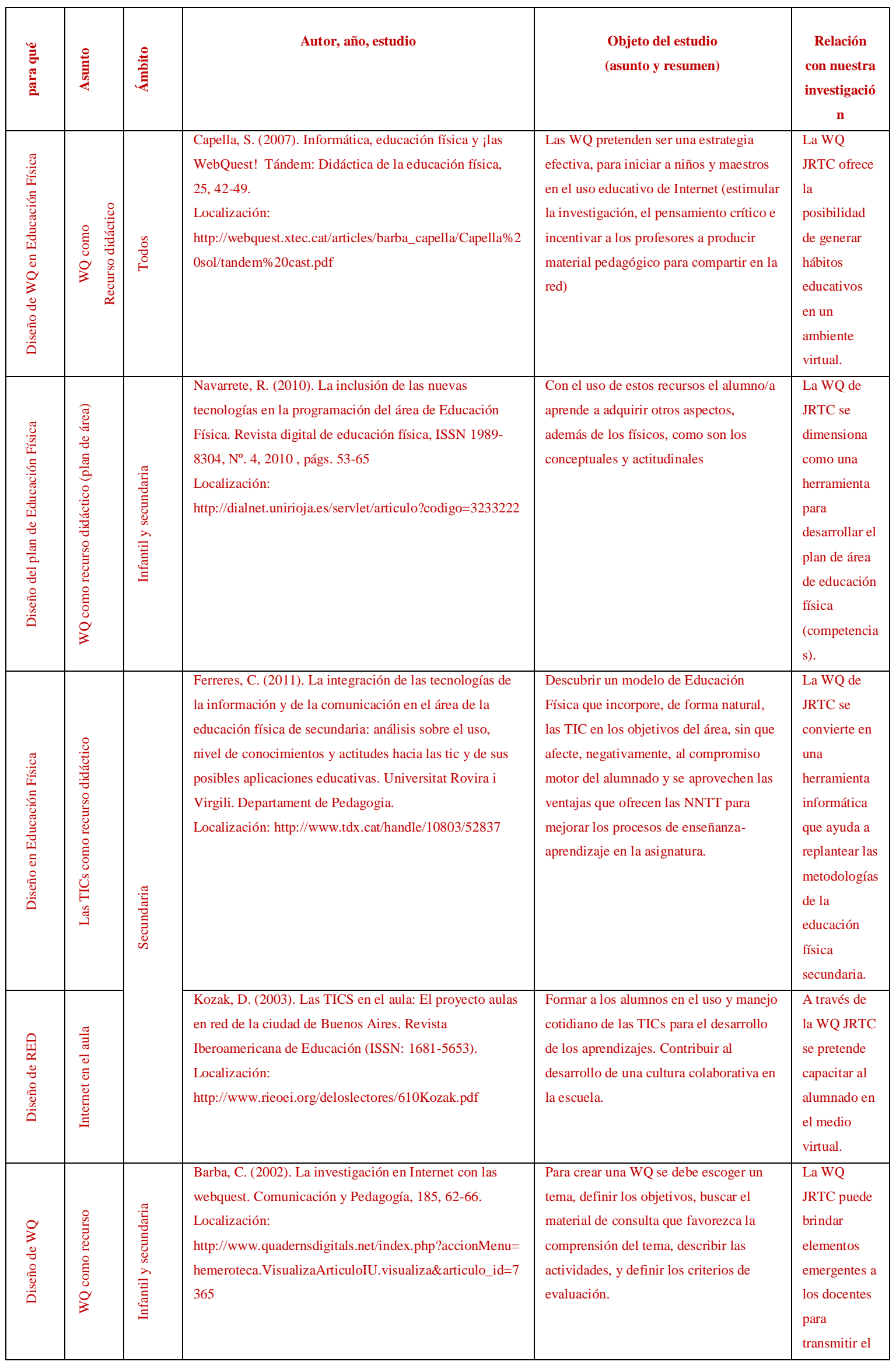

Sistematización, documentación y divulgación virtual de los Juegos Recreativos... 


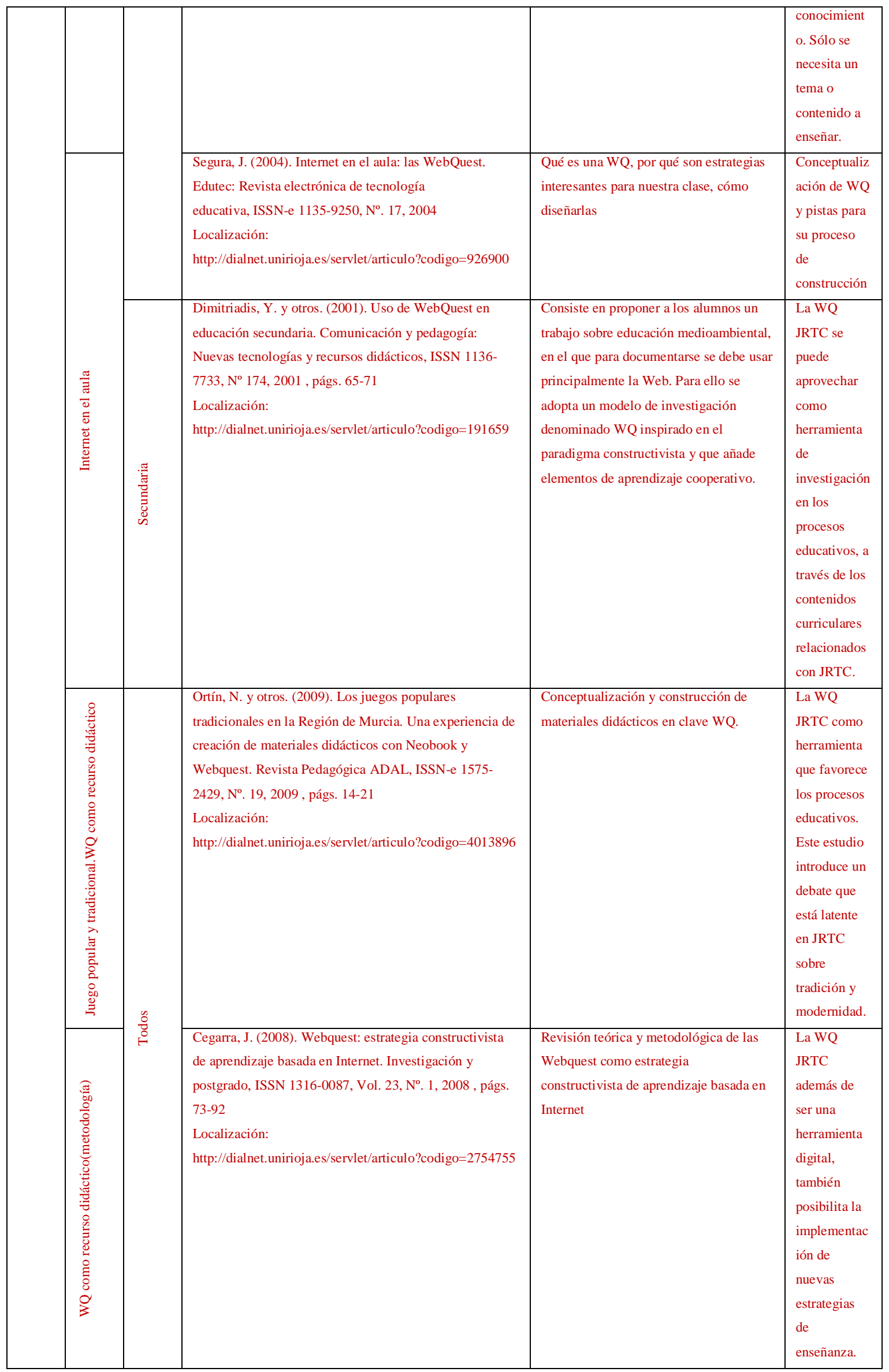




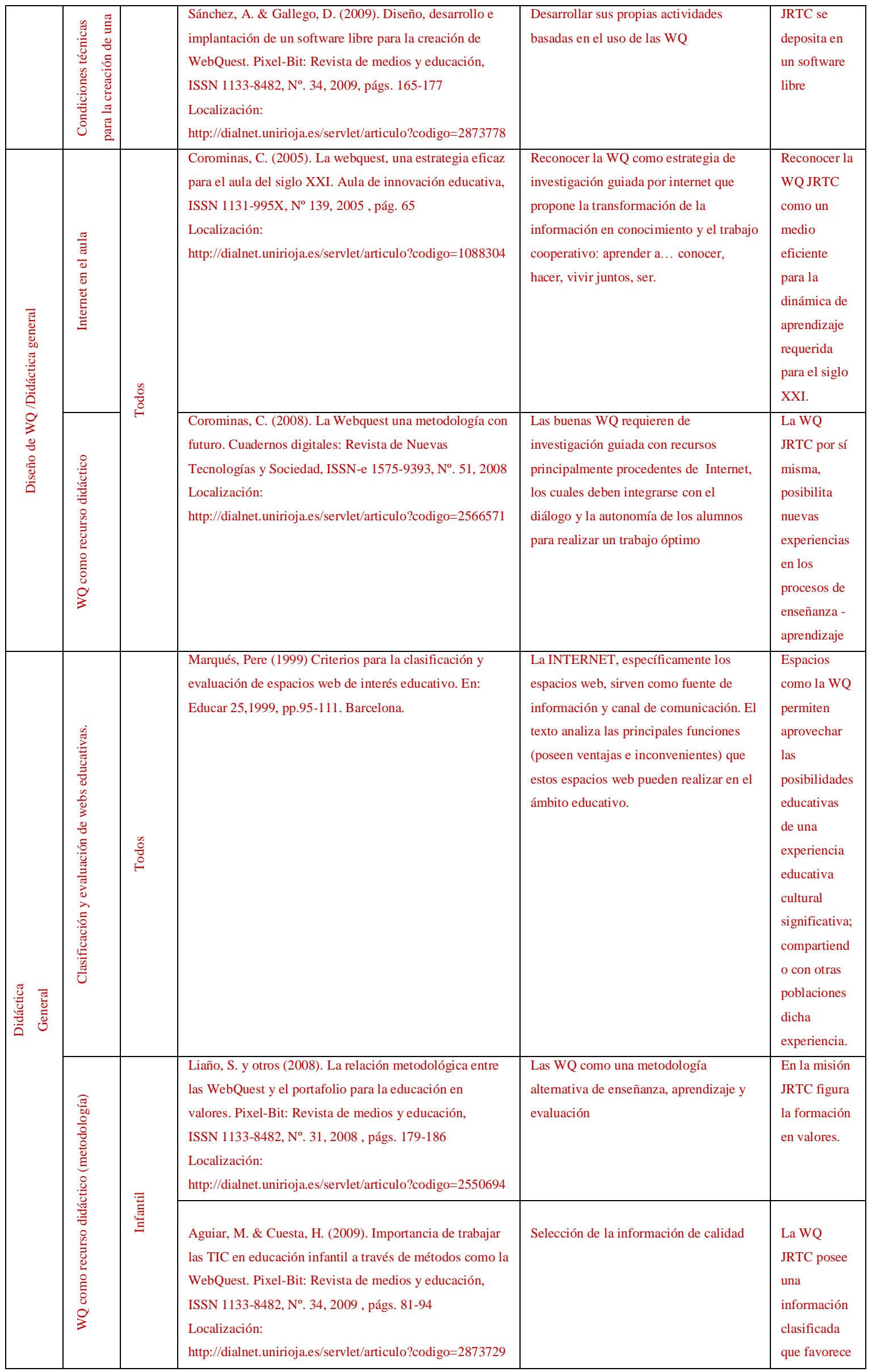




\begin{tabular}{|c|c|c|c|c|}
\hline & & & & $\begin{array}{l}\text { el uso de la } \\
\text { información. }\end{array}$ \\
\hline \multirow{3}{*}{ 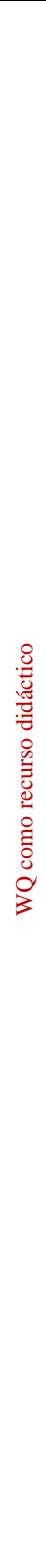 } & & $\begin{array}{l}\text { Martínez, A. (2007). Metodología y tecnologías de la } \\
\text { información y comunicación: WebQuest. Alicante }\end{array}$ & $\begin{array}{l}\text { En esta ponencia se plantea un modelo } \\
\text { de aprendizaje que combina los } \\
\text { pequeños proyectos de investigación en } \\
\text { Educación Infantil con la utilización de } \\
\text { recursos digitales, WQ. }\end{array}$ & $\begin{array}{l}\text { A través de } \\
\text { este modelo } \\
\text { de } \\
\text { aprendizaje, } \\
\text { las personas } \\
\text { pueden } \\
\text { acceder a } \\
\text { estos } \\
\text { contenidos y } \\
\text { adaptarlos } \\
\text { según sus } \\
\text { necesidades. }\end{array}$ \\
\hline & 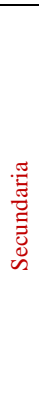 & $\begin{array}{l}\text { Antolín, J. (2008). WebQuest, estrategia didáctica basada } \\
\text { en Internet. Hekademus: Revista Científica de la } \\
\text { Fundación Iberoamericana para la Excelencia } \\
\text { Educativa, ISSN-e 2027-1824, N. 1, } 2008 \text {, págs. 6-7 } \\
\text { Localización: } \\
\text { http://dialnet.unirioja.es/servlet/articulo?codigo=3287085 }\end{array}$ & $\begin{array}{l}\text { Utilizar estrategias didácticas que } \\
\text { faciliten el aprendizaje de los alumnos y } \\
\text { a su vez, desarrollar oportunidades de } \\
\text { aprendizaje en los campos donde se } \\
\text { desempeña la comunidad educativa. }\end{array}$ & $\begin{array}{l}\text { Con la WQ } \\
\text { JRTC, se } \\
\text { dispone al } \\
\text { alumno hacia } \\
\text { un ambiente } \\
\text { de trabajo } \\
\text { cooperativo } \\
\text { con } \\
\text { objetivos } \\
\text { definidos. }\end{array}$ \\
\hline & & $\begin{array}{l}\text { Fierro, J. (2005). La oportunidad WebQuest. Revista } \\
\text { DIM: Didáctica, Innovación y Multimedia, ISSN-e 1699- } \\
3748, N^{\circ} .2,2005 \\
\text { Localización: } \\
\text { http://dialnet.unirioja.es/servlet/articulo?codigo=1400295 }\end{array}$ & $\begin{array}{l}\text { Trabajo cooperativo, creatividad y } \\
\text { destino social de la WQ }\end{array}$ & $\begin{array}{l}\text { La WQ } \\
\text { JRTC es una } \\
\text { oportunidad } \\
\text { para que los } \\
\text { organizadore } \\
\text { s proyecten } \\
\text { el destino } \\
\text { social de los } \\
\text { JRTC. }\end{array}$ \\
\hline 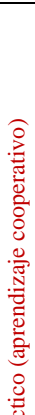 & 吾 & $\begin{array}{l}\text { Quintana, J., \& Higueras, E. (2009). Las Web-quests, una } \\
\text { metodología de aprendizaje cooperativo, basada en el } \\
\text { acceso, el manejo y el uso de información en la red. } \\
\text { Barcelona: Octaedro, Universitat de Barcelona/Institut de } \\
\text { Ciéncies de l'Educació. } \\
\text { Localización: } \\
\text { http://www.octaedro.com/ice/pdf/11CUADERNO.pdf }\end{array}$ & $\begin{array}{l}\text { Las WQ son propuestas de trabajo para } \\
\text { estudiantes en las que, a partir de la } \\
\text { presentación de un tema, y su contexto, } \\
\text { se propone la realización de un trabajo o } \\
\text { proyecto en grupo (generalmente termina } \\
\text { en una exposición pública), usando } \\
\text { información básicamente de Internet, y } \\
\text { seleccionada para garantizar la calidad. }\end{array}$ & $\begin{array}{l}\text { El propósito } \\
\text { es que } \\
\text { cualquier } \\
\text { docente } \\
\text { amplíe su } \\
\text { abanico de } \\
\text { metodologías } \\
\text {, más allá de } \\
\text { su } \\
\text { asignatura. }\end{array}$ \\
\hline
\end{tabular}




\begin{tabular}{|c|c|c|c|c|c|}
\hline & & 䔍 & $\begin{array}{l}\text { Capllonch, M. (2005). Las tecnologías de la información } \\
\text { y la comunicación en la Educación Física de primaria: } \\
\text { estudio sobre sus posibilidades educativas. Tesis doctoral. } \\
\text { Barcelona, Universidad de Barcelona. } \\
\text { Localización: } \\
\text { http://www.tesisenred.net/handle/10803/2907 }\end{array}$ & $\begin{array}{l}\text { Descubrir el tratamiento que deben tener } \\
\text { las TIC en la Educación Física escolar, } \\
\text { tanto desde la perspectiva del } \\
\text { profesorado como del alumnado }\end{array}$ & $\begin{array}{l}\text { Capacitar } \\
\text { alumnos y } \\
\text { docentes en } \\
\text { cuanto al uso } \\
\text { de las TICs. }\end{array}$ \\
\hline & 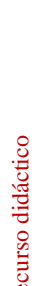 & & $\begin{array}{l}\text { Ambrós, Q. (2012). Las tecnologías del aprendizaje y el } \\
\text { conocimiento (TAC) en la educación física, la WQ como } \\
\text { recurso didáctico. Apunts: Educación física y deportes, } \\
\text { ISSN 1577-4015, N } 109,2012 \text {, págs. } 44-53 \\
\text { Localización: } \\
\text { http://dialnet.unirioja.es/servlet/articulo?codigo=4076222 }\end{array}$ & $\begin{array}{l}\text { Ventajas de la utilización de las TAC en } \\
\text { un proceso autónomo de aprendizaje en } \\
\text { las relaciones interpersonales entre } \\
\text { alumnos }\end{array}$ & $\begin{array}{l}\text { La WQ } \\
\text { puede } \\
\text { favorecer el } \\
\text { aprendizaje } \\
\text { autónomo de } \\
\text { los } \\
\text { estudiantes }\end{array}$ \\
\hline$\frac{\widetilde{n}}{\frac{\pi}{2}}$ & $\begin{array}{l}0 \\
0 \\
0 \\
0 \\
0 \\
3\end{array}$ & 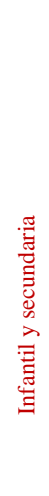 & $\begin{array}{l}\text { Guimaraes, R. (2009). Utilización de una webquest como } \\
\text { recurso didáctico para intervenir en casos de bullying } \\
\text { desde el área de Educación Física. Revista Educación } \\
\text { física y deporte, n. 28-1, 41-51, 2009, Funámbulos } \\
\text { Editores. } \\
\text { Localización: } \\
\text { http://aprendeenlinea.udea.edu.co/revistas/index.php/educ } \\
\text { acionfisicaydeporte/article/view/2995/2718 }\end{array}$ & $\begin{array}{l}\text { Considerar la WQ como un importante } \\
\text { recurso didáctico de apoyo a la } \\
\text { enseñanza en el área de Educación Física }\end{array}$ & $\begin{array}{l}\text { Reconocer } \\
\text { las } \\
\text { propiedades } \\
\text { de la WQ } \\
\text { JRTC. En } \\
\text { este caso, el } \\
\text { uso } \\
\text { comunicacio } \\
\text { nal ante } \\
\text { situaciones } \\
\text { de acoso } \\
\text { escolar. }\end{array}$ \\
\hline 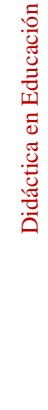 & 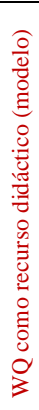 & & $\begin{array}{l}\text { Fernández, J. (2007). La aplicación de las TICs en el área } \\
\text { de Educación Física a través del modelo didáctico de la } \\
\text { Webquest. Revista Iberoamericana de Educación, ISSN- } \\
\text { e 1681-5653, Vol. 44, N. 5, } 2007 \\
\text { Localización: } \\
\text { http://dialnet.unirioja.es/servlet/articulo?codigo=2471792 }\end{array}$ & $\begin{array}{l}\text { Inclusión en el área de Educación Física, } \\
\text { a través del modelo didáctico de las WQ }\end{array}$ & $\begin{array}{l}\text { JRTC puede } \\
\text { desplegarse a } \\
\text { través del } \\
\text { modelo } \\
\text { didáctico } \\
\text { WQ para } \\
\text { usos de la } \\
\text { clase de } \\
\text { educación } \\
\text { física. }\end{array}$ \\
\hline & 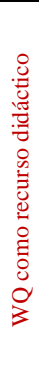 & 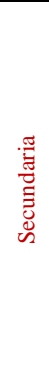 & $\begin{array}{l}\text { Ramos, L. (2009). Utilización de las webquest como } \\
\text { recurso educativo en educación física. Quaderns digitals: } \\
\text { Revista de Nuevas Tecnologías y Sociedad, ISSN- } \\
\text { e 1575-9393, No. 59, } 2009 \\
\text { Localización: } \\
\text { http://dialnet.unirioja.es/servlet/articulo?codigo=3037325 }\end{array}$ & $\begin{array}{l}\text { Hacer un análisis sobre el uso de Internet } \\
\text { en la materia de Educación Física, } \\
\text { basado en un modelo de investigación } \\
\text { denominado WQ. }\end{array}$ & $\begin{array}{l}\text { Con esta } \\
\text { herramienta } \\
\text { virtual, se } \\
\text { busca que el } \\
\text { alumno sea } \\
\text { el } \\
\text { protagonista } \\
\text { de su propio } \\
\text { aprendizaje. }\end{array}$ \\
\hline & 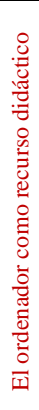 & $\begin{array}{l}\frac{\pi}{0} \\
\stackrel{0}{0} \\
\stackrel{0}{0}\end{array}$ & $\begin{array}{l}\text { Gordejo, F. J. (2004) El ordenador como recurso de } \\
\text { enseñanza-aprendizaje en Educación Física. Efedeportes } \\
\text { Revista Digital, } 77 . \\
\text { Localización: } \\
\text { http://www.efdeportes.com/efd77/ordenad.htm }\end{array}$ & $\begin{array}{l}\text { Los profesores tenemos a nuestro } \\
\text { alcance un valioso medio. Esta situación } \\
\text { nos impone la actualización de nuestra } \\
\text { metodología de enseñanza. }\end{array}$ & $\begin{array}{l}\text { La WQ } \\
\text { JRTC le } \\
\text { permite al } \\
\text { profesor de } \\
\text { Educación } \\
\text { Física, } \\
\text { descubrir las } \\
\text { posibilidades } \\
\text { que ofrecen } \\
\text { las NNTT. }\end{array}$ \\
\hline
\end{tabular}




\begin{tabular}{|c|c|c|c|c|c|}
\hline$\stackrel{\mathscr{0}}{0}$ & 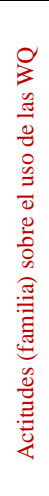 & 䔍 & $\begin{array}{l}\text { Goig, R. (2010). Opiniones y actitudes de las familias } \\
\text { ante el uso de la WebQuest en la etapa de Educación } \\
\text { Infantil. Revista DIM: Didáctica, Innovación y } \\
\text { Multimedia, ISSN-e 1699-3748, N } .18,2010 \\
\text { Localización: } \\
\text { http://dialnet.unirioja.es/servlet/articulo?codigo=3671487 }\end{array}$ & $\begin{array}{l}\text { Detectar la actitud de las familias hacia } \\
\text { el uso de la WQ }\end{array}$ & $\begin{array}{l}\text { Este estudio } \\
\text { hace pensar } \\
\text { en la } \\
\text { necesidad de } \\
\text { indagar } \\
\text { acerca de las } \\
\text { actitudes y } \\
\text { opiniones de } \\
\text { las familias } \\
\text { frente al uso } \\
\text { de la WQ de } \\
\text { JRTC. }\end{array}$ \\
\hline
\end{tabular}




\section{MARCO REFERENCIAL}

\subsection{Webquest Juegos de la Calle}

Los nativos predican que "Los Juegos Recreativos Tradicionales de la Calle son de Caldas para el mundo", pero el mundo no los conoce. Estos a juicio de los caldeños 5 , "al quedarse en el activismo, son poco conocidos por fuera de la parroquia..."; los resultados de la investigación de base permiten atrever que requieren de organización (análisis y categorización textual) del material existente, de sistematización e investigación. Hay consenso entre nativos e investigadores, se hace imperativa la necesidad de crear una herramienta de divulgación que permita a través de la INTERNET: (1) El mejoramiento del uso educativo - comunicacional de los JRTC (interactivo, educativo, informativo, enlace, depósito, gestión, retroalimentación, evaluación), (2) El reconocimiento local, regional, nacional e internacional; proceso que permita una presentación de los JRTC a la comunidad académica, política, turística, comercial, educativa. Sugiere Marqués, P. (1999, pp. 95-96) que en la sociedad de la información, los proyectos educativos, las instituciones educativas,

como elementos culturizantes [es el caso de JRTC] se quedan pequeños...INTERNET constituye un canal de comunicación a escala mundial, cómodo, versátil y barato que facilita la comunicación..., permite compartir y debatir ideas y facilita el trabajo cooperativo y la difusión de las creaciones...

Es la INTERNET una herramienta potente que permite abrir puertas a experiencias educativas o culturales significativas que se pierden en un activismo de alcance local.

La Webquest, es una dirección global de documentos en Internet, para Dodge (2002) una Webquest consiste en una

actividad de indagación/investigación enfocada a que los estudiantes obtengan toda o la mayor parte de la información que van a utilizar de recursos existentes en Internet.

En este caso para este investigador la WQ es una herramienta que se basa en una información proveniente de internet usada por el investigador. Pensando en una

\footnotetext{
${ }^{5}$ Ver entrevista a Tiberio Amaya, profesor de Caldas. Investigación CICIDEP "Documentación, sistematización e interpretación de buenas prácticas pedagógicas en los procesos de educación del cuerpo (capítulo Colombia)". Informe final de proyecto de Extensión 2011.
} 
aplicación educativa. Para el caso de Juegos de La Calle, es una hoja de trabajo URL que facilita el proceso educativo- comunicacional de los JRTC.

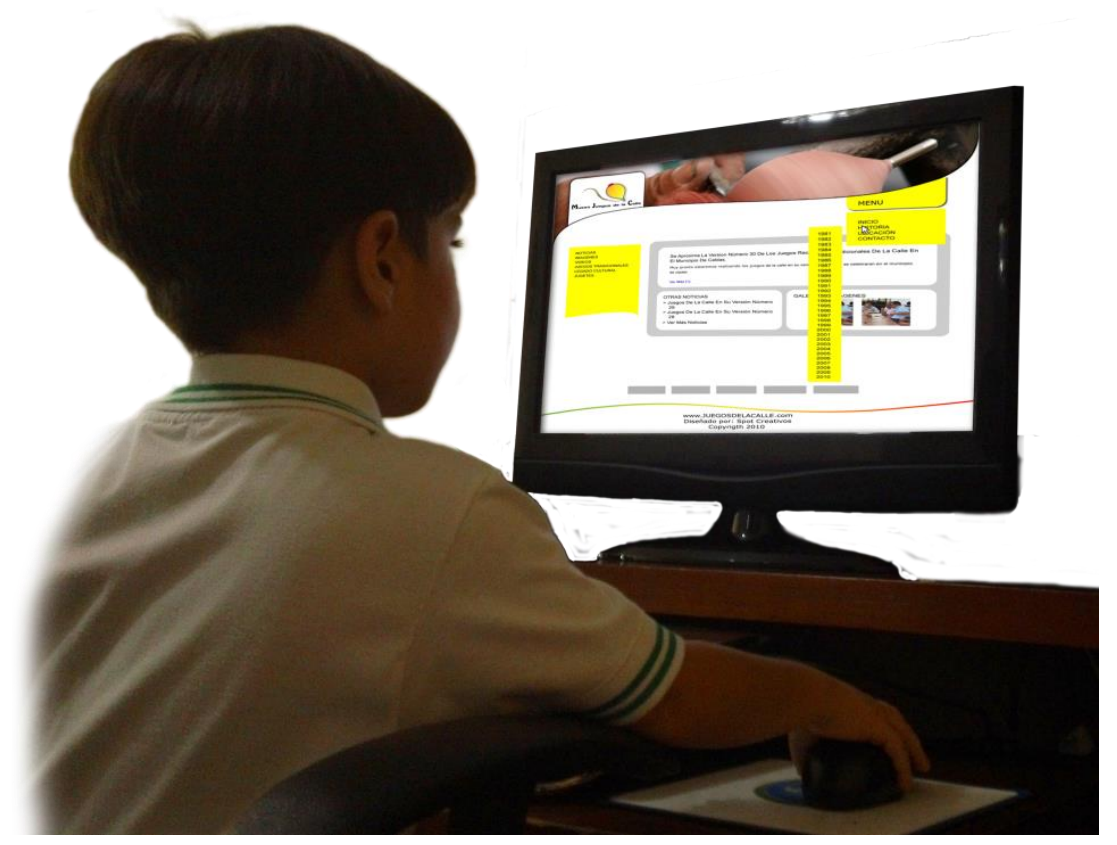

Fotografía 2. Entrando a la Webquest de JRTC (Fotomontaje).

Propiedades de la webquest que requieren los JRTC:

- Interactiva, propicia la comunicación entre personas interesadas sobre los juegos y sobre asuntos relacionados con el juego recreativo tradicional), intercambio de experiencias.

- Informativa, sirve como depósito de textos (escritos, video-gráficos, fotográficos, etc.), programaciones, convocatorias, actas, memorias, invitaciones, cartas. Expone muestras, resultados de eventos...

- Educativa (capacita, instruye, brinda elementos didácticos acerca de la enseñanza de los juegos, divulga cartillas, manuales, currículos, manifiestos pedagógicos; todos estos relacionados con JRTC), permite el trabajo colaborativo entre actores interesados en el juego.

- Recipiente interactivo para el producto investigativo sobre JRTC, Juego tradicional, juego, políticas sobre juego, permite guardar deposito de textos. 
- Requiere recursos computacionales, acceso a internet, capacitación básica sobre internet para los usuarios, retroalimentación, sostenimiento.

- Enlaza sitios de interés, actores y agencias (INDER, INDEPORTES, COLDEPORTES, COI, INDEC...) de juegos, facilita búsquedas especializadas sobre el tema.

- Administrativas, permite actividades de gestión de recursos, de información, cultural, social, recreo- deportiva.

\subsection{Juego}

Gómez (2006), pionero de los JRTC, plantea que el hombre a través de la historia ha sentido la necesidad de ejecutar actividades lúdicas en el largo periodo de su infancia; actividad que según él cumple con una función biológica con alto contenido cultural. Huizinga citado por Torres \& Torres (2007), sostendrá que el juego es una acción voluntaria realizada en ciertos límites fijos de tiempo y lugar, con una regla libremente consentida pero absolutamente imperiosa, provista de un fin en sí, acompañada de una sensación de tensión y de jubilo, y de la conciencia de ser de un modo u otro que el de la vida real.

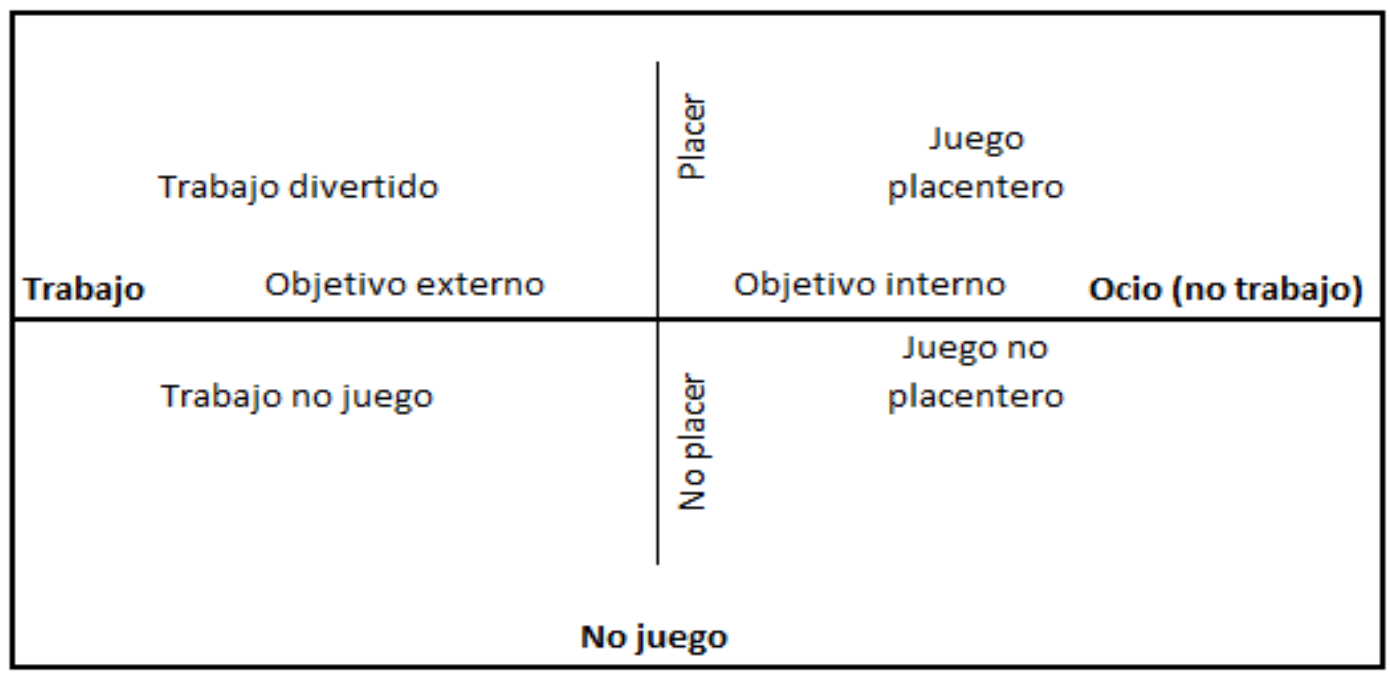

Gráfica 1. Blanchard - Cheska, (1986)

La gráfica 1 permite considerar el sistema de relaciones entre las diferentes dimensiones del juego según su estado y objetivo. Podemos evidenciar que JRTC refleja en su lógica constitutiva tal sistema de relaciones.

Es preciso hacer una claridad entre lo que es juego y deporte. Olivera (1989) plantea que el juego es inherente a la formación de la cultura del ser humano; aclara que todo el 
deporte pertenece al juego; pero no todo juego es deporte. En investigaciones del grupo PES $^{6}$, se ha ubicado un debate en Caldas sobre la naturaleza de los JRTC "al decir de un sector significativo de sus defensores es un ritual colectivo que sobrepasa las justas deportivas tradicionales" (Moreno, 2013, pág 22); unos lo consideran deporte; otros lo elevan a práctica educativa y/o recreativa; otros lo nombran como juego deportivo.

\subsection{Juego recreativo}

Retomando a Gómez (2006), una verdadera recreación debe contener tres elementos fundamentales: libertad de decisión, creatividad y satisfacción personal. La gráfica 1, da cuenta de lo que representa el juego para un individuo de acuerdo a la posición y finalidad que éste le atribuya. En ese sentido JRTC se concibe como una actividad donde la mejor retribución que se ha de esperar es el placer y el divertimento. Hay competencia pero parece que se impone la lógica del juego antes que la lógica del campeonato. Es evidente en la base documental que la lógica competitiva-deportiva viene ganando espacio en los últimos años a la lógica del juego-recreativo; la lógica del festival y del encuentro sede ante las transferencias de los campeonatos y la representación institucional intencionada por los puntos, el trofeo y el campeonar. El juego recreativo tiende a ceder terreno ante la prevalencia social de una competición avasallante en las diferentes dimensiones de la vida y de la interacción social.

\subsection{Juego tradicional}

Para Castro, Amador \& Álamo (2005), los juegos tradicionales son prácticas que se aprenden por trasmisión oral, la observación/imitación y sobre todo por la propia acción motriz de jugar; constituyendo un extraordinario legado de costumbres y aprendizajes.

En este orden de ideas, parece que, hablar de juego tradicional en Caldas implica pensar en procesos, relaciones y organizaciones sociales que se han ido consolidando alrededor de esta práctica social y ludo recreativa a lo largo de treinta y tres años. Se juega evocando prácticas ancestrales de origen local, nacional e internacional, se juegan esencialmente los juegos que hacen parte de una tradición que ya no se reduce a la experiencia local, juegos que llegaron de ultramar y que parecen haberse impuesto sobre los juegos que componían la tradición autóctona, nativa. Hay esfuerzos significativos

\footnotetext{
${ }^{6}$ Véase, Moreno, W. y otros 2009-2011 y Moreno, W. y otros 2013
} 
especialmente impulsados por comunidades bolivianas y argentinas por recuperar el juego tradicional autóctono ${ }^{7}$.

\subsection{Juegos de la calle}

En nuestro contexto llamamos juegos de la calle a las actividades con las características que encierran la palabra "juego" definida anteriormente, donde el escenario por excelencia, son las calles, que generalmente poseen una estrecha relación con el juego tradicional. El juego callejero puede ser institucionalizado (caso JRTC), medianamente institucionalizado (caso el día de la pereza en Itagüí-Antioquia) o no institucionalizado (caso los juegos espontáneos que niños y jóvenes despliegan en las calles de las grandes ciudades especialmente en sus sectores más populares ${ }^{8}$ ). Los procesos de urbanización avasallante como se evidencia en el estudio de López y otros (2012) dan al traste con la experiencia lúdica callejera urbana. Este estudio se encuentra con una práctica callejera informal, el Picao que se despliega

dramáticamente en una ciudad (Medellín) que parece redefinir el espacio público recreativo bajo los criterios utilitaristas y consumistas de un deporte domesticado y domesticante que se impone de espaldas a las tradiciones y vivencias ludo recreativas que hacen parte de la experiencia y las necesidades proyectadas por la propia ciudadanía marginada

El juego callejero pierde ante los procesos de domesticación institucional de las prácticas lúdicas urbanas por parte de los urbanistas grises.

\subsection{Juegos Recreativos Tradicionales de la Calle}

Gómez (2008, pp. 24) se remonta a la década delos 80's, donde tres estudiantes universitarios iniciaron los juegos en la escuela Pedro Luis Álvarez Correa por dos razones: la primera de orden lúdico, porque es una práctica muy antigua; y la segunda, "de la calle", porque es el escenario por excelencia donde se desarrolla la vida social pública de todas las culturas del mundo. Con los años, los JRTC se han convertido en política pública municipal recibiendo el respaldo de la Institución Educativa Caldeña.

\footnotetext{
${ }^{7}$ Véase Ferrarese, 2013

${ }^{8}$ Estudios realizados en la comuna 16 de Medellín por el grupo PES (véase, Moreno y otros 2014), encontraron que en las calles de las localidades de Belén se despliegan una diversidad de prácticas ludocorporales no mediadas por procesos de institucionalización tales como: Trompo, carro de rodillos, picao, gimnasio rural, piscina inflable, parkour, trote con o sin mascotas, caminata, Bike Trial callejero, down hill, golf callejero, parapentismo, yeimi, bota tarro, juegos de esquina: cartas, dominó, dados.
} 


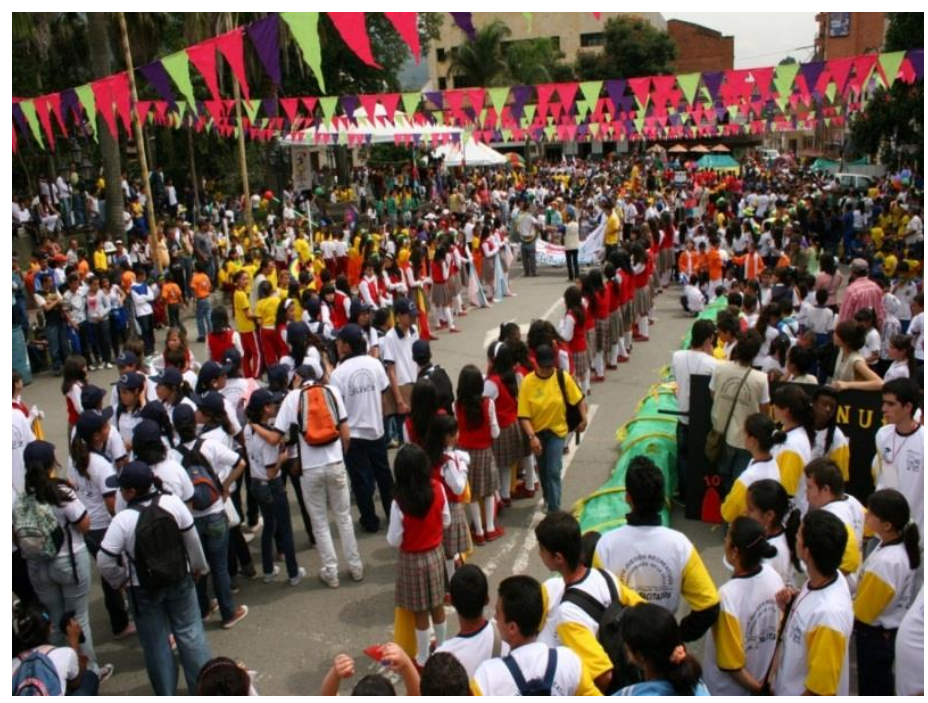

Fotografía 3. JRTC. Patrimonio histórico caldeño

Los JRTC se desarrollan en tres fases: 1) Iniciatoria que comprende la parte informativa y motivacional hacia los estudiantes, 2) Intramural, a través de competiciones que se dan al interior de la escuela, y, 3) Extramural, con competiciones municipales y nacionales donde la escuela y la comunidad se encuentran en la calle. Siendo esta, según Humberto Gómez (2006, pp. 53) “el escenario central de su nacimiento, realización y evolución a través de las diferentes etnias de la humanidad".

Los Juegos de la calle se desarrollan a través de la ejecución de una serie de juegos de carácter tradicional que son "entrenados" en las instituciones educativas, generalmente en el marco de la clase de educación física. Los Juegos convocan a todas las oficinas del gobierno municipal, a los entes deportivo recreativo departamental y nacional. La mesa departamental de Educación Física y una organización interinstitucional, son los responsables de la organización de éstas justas deportivo-recreativas.

\section{METODOLOGÍA}

Este estudio le apuesta a una solución comunitaria, se constituye en un proceso que va de la sistematización de la documentación histórica de JRTC hasta la configuración de una herramienta virtual que aloje dicha información en un formato propicio para la formación comunitaria.

En este estudio entendemos por sistematización, de acuerdo con Ghiso (2001),

el proceso de recuperación, tematización y apropiación de una practica formativa determinada, que al relacionar sistémica e históricamente sus componentes teórico-prácticos, permite a los sujetos comprender y explicar 
los contextos, sentidos, fundamentos, lógicas y aspectos problemáticos que presenta la experiencia, con el fin de transformar y cualificar la comprensión, experimentación y expresión de las propuestas educativas de carácter comunitario

Metodológicamente, como se puede apreciar en la gráfica 2, se adoptan procesos propios de la investigación acción: se ubica un problema comunitario, se definen posibles soluciones, se selecciona una solución, se pone en acción, se evalúa la primera ejecución (primera espiral constructiva). De ser necesario, se definen nuevas espirales. Es junto a la comunidad caldeña que, culminando la fase de experimentación básica, se plantean los ajustes y redireccionamientos que requiera la herramienta divulgativa (webquest JRTC) que se experimenta desde una base virtual experimental depositada en la web ${ }^{9}$.

Identificación de Necesidad y Problematización (Pregunta orientadora), contacto con las instituciones

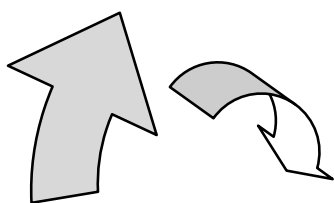

Observación, evaluación de resultados y replanteamiento participado (Inicio de nueva espiral

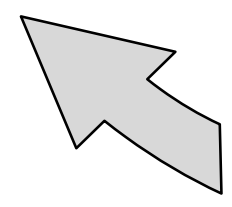

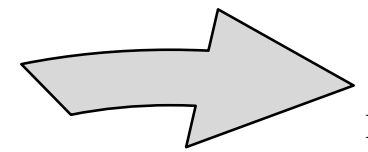

Establecimiento de un plan acción investigativo

Estudio de diferentes herramientas virtuales.

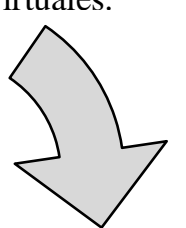

Puesta en acción.

(Experimentando) Selección y adecuación de la herramienta virtual.

Clasificación y enmarcación del material y del producto histórico JRTC. Alojamiento en la herramienta virtual del producto de la sistematización

\section{Puesta en acción}

(Desarrollo de la propuesta)

Apertura pública de la herramienta en forma experimental y en forma estable.

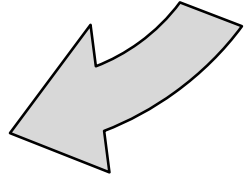

Gráfica 2. Proceso en la investigación -acción (fases).

El proyecto consta de dos fases: la primera denominada fase de sistematización, en ella se recuperó y organizó (clasificar, enmarcar y editar) la documentación histórica dispersa, registrada desde el año 1981 hasta el año de 2014. Este material se hace visible en la red a través de la webquest; herramienta que le permite al usuario interactuar con el administrador y con los demás usuarios de la web.

\footnotetext{
${ }^{9}$ Véase, juegosdecaldas.com
} 


\section{Fuentes recolección de la información}

Discursos de los actores de JRTC registrados en diferentes textos (actas, manuales, prensa, políticas y normas). Registros fotográficos y video gráficos de JRTC

\section{Técnicas de recolección de la información}

Registro fotográfico y video gráfico (de las diferentes fases de los JRTC y de la documentación en archivo). Análisis de documentos y artefactos (trofeos, juguetes, retablos, afiches).

\section{Instrumentos}

La matriz de la sistematización:

Para el registro documental de las fotografías recopiladas por el grupo a través de la intervención, se pensó en varios formatos y modelos que permitieran detallar minuciosamente cada imagen. En el proceso de elaboración hubo varios cambios que nos llevó al final a optar por una estructura en formato Excel (ver gráfica .3), que detallara las características de la imagen en aspectos relacionados con el autor, la fecha; la descripción del evento,el lugar, losparticipantes y el tipo de acciones ejecutadas y manifiestas en cada fotografía, para que al final cada imagen por si sola permitiera su descripción y a su vez la visualización de estas prácticas que hacen parte de la cultura de esta comunidad Antioqueña. 


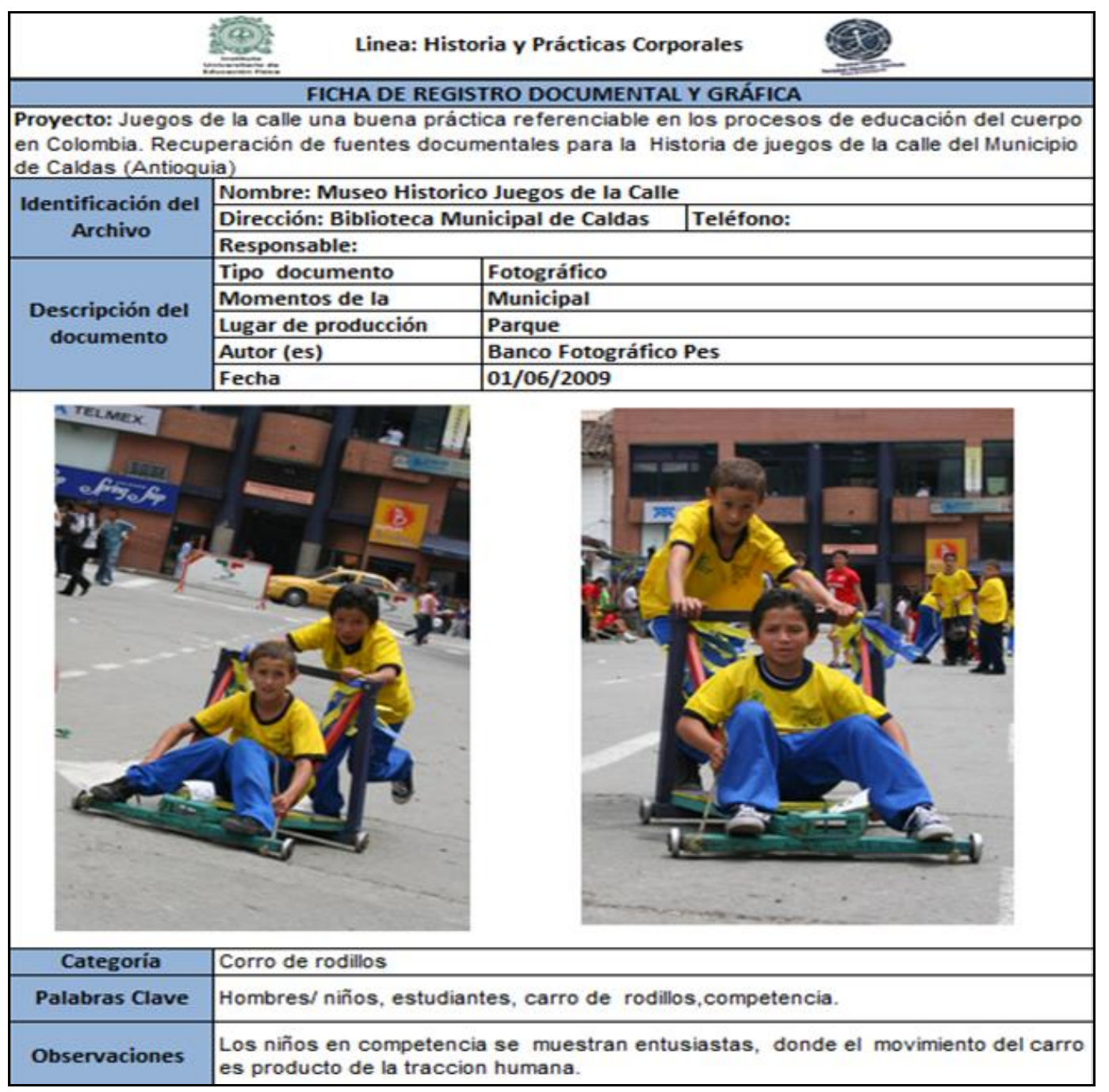

Gráfica 3. La matriz de la sistematización

\section{La Webquest}

Según Dodge (2002) la WQ consiste en una

"actividad de indagación/investigación enfocada a que la comunidad interesada obtenga toda o la mayor parte de la información que van a utilizar de recursos existentes en Internet".

La webquest además de representar un interesante medio para la divulgación y la investigación y creación participada, también es un medio de alto potencial para los procesos de enseñanza; JRTC se convierte en selección cultural curricularizable, forma potente de divulgar y desarrollar el potencial educador de los JRTC.

Ha medida que el proceso constructivo ha avanzado la webquest JRTC se plantea como una hoja de trabajo URL que contiene en forma rigurosa el patrimonio histórico de Juegos de la Calle sistematizado a partir de diferentes nodos y unidades temáticas; 
disposición que favorece el acceso de la comunidad academice nacional e internacional a la cultura JRTC.

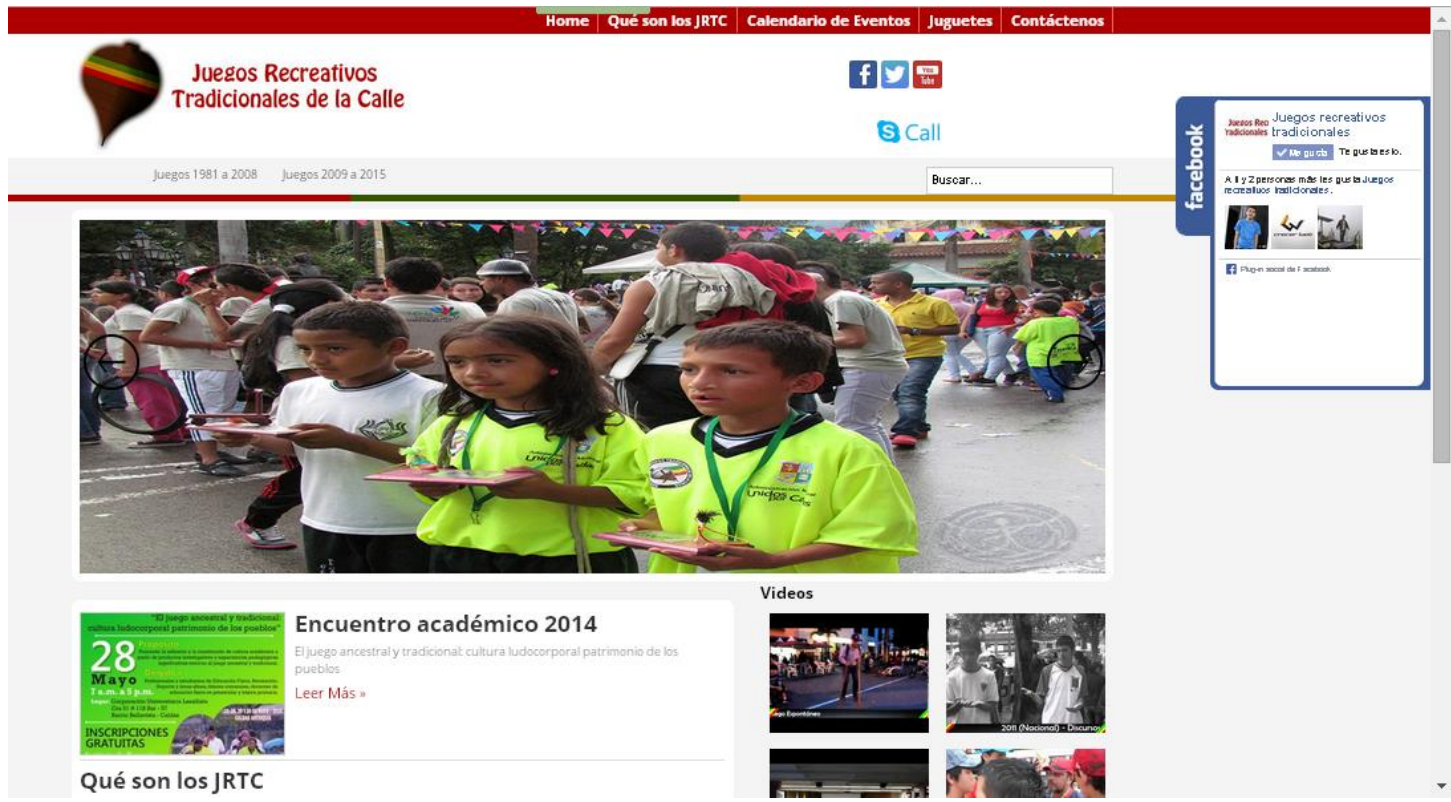

Gráfica 4. La Web Quest JRTC.

\section{Resultados}

A través de un ejercicio riguroso del equipo investigativo, se ha logrado una sistematización de la información histórica de los JRTC (producción textual entre 19812014): Documentos históricos (actas, manifiestos, comunicados, invitaciones, manuales, libros, fotografías, diarios, presupuestos, proyectos, cartas, fragmentos de periódicos alusivos al evento, comunicaciones interinstitucionales, videos). Esta base documental (Patrimonio histórico de Caldas) ha sido alojada en la WebQuest JRTC propia, conectada a la web institucional del municipio de Caldas. Esta herramienta mediática permite la divulgación del patrimonio cultural JRTC; instituciones de educación formal y no formal, deportiva y recreativa, ciudanía en general tienen acceso virtual a través de una dirección web.

\section{Referencias bibliográficas}

Castro, U., Amador, F. \& Álamo, J. (2005): Juegos tradicionales: Universal Cultural del Siglo XXI. Excemo.

Blanchard \&Cheska, A. (1986): Antropología del deporte. Bellaterra. Barcelona. 
Dodge, B. (2002): Cinco Reglas para Escribir una Fabulosa WebQuest. http://www.eduteka.org/profeinvitad.php3?ProfInvID=0010

Dodge, B. Paladín del Aprendizaje Basado en Internet. En EDUTEKA (2002). http://www.eduteka.org/reportaje.php3?ReportID=0011

Demetrio, B. (1999): Acerca de la fotografía etnográfica. En Gazeta de Antropología No 15, Art.11.

Ferrarese, S. (2013): El derecho a jugar: El juego étnico en una sociedad multiétnica un desafío para el aula y la sociedad. Disertación Universitaria de Educación Física. Evento académico organizado por el Grupo de investigación PES de la Universidad de Antioquia. Mes de Junio.

Gómez, H. (2006): Juegos Recreativos Tradicionales de la Calle: una herramienta pedagógica. Medellín: L. Vieco e Hijas Ltda.

Ghiso, A. (2001): Sistematización de experiencias en Educación popular. Memorias Foro: Los contextos Actuales de la Educación Popular. Medellín.

Jara, O. (1996): Tres posibilidades de sistematización: comprensión, aprendizaje y teorización. En Aportes 44. Sistematización de experiencias Búsquedas recientes. Dimensión Educativa Pág. 15

Las WebQuest y el Uso de la Información. En EDUTEKA.

http://www.eduteka.org/comenedit.php3?ComEdID=0010

López J., Toro C. \& Moreno W. (2012): Picao, drama emblemático de una práctica ludo deportiva que refleja los proceso de redefinición de la ciudad y de las culturas de los jóvenes que la habitan. Revista Educación Física y deporte Vol. 31.1

Luna, J. (2009): Foto-etnografía llevada a cabo por personas en situación de pobreza en la frontera norte de México. En Forum: Qualitative Social Research Sozialforschung, Vol. 10, $\mathrm{N}^{\mathrm{o}}$ 2, Art. 35.

Marqués, P. (1999): Criterios para la clasificación y evaluación de espacios web de interés educativo. En Educar 25,1999, pp.95-111.

Melleiro, M \& Gualda, R (2005): La foto voz como una estrategia para la recolección de datos en una investigación etnográfica. En Ciencia y enfermería XI (1). 
Moreno, W; Pulido, S; Gaviria, N; Mendoza, J; Cardona, H; García, Saldarriaga, J; Escobar, J; Díaz, N; Mejía, A; Herrera, M.I.; D; Hincapié. (2014): Las prácticas ludocorporales como dispositivos configuradores del entramado social de la ciudad de Medellín 2013-2014. Informe de investigación. Universidad de Antioquia (CICIDEP), INDER Medellín (en imprenta).

Moreno, W. \& Betancur, G. (2008): Los juegos de la calle, un reflejo de los límites de la escolarización, un horizonte para la formación en el libre juego. En Revista Educación Física y Deporte, Vol. 27, No. 1, Universidad de Antioquia, Medellín.

Moreno, W. y otros (2009-2011): Documentación, sistematización e interpretación de buenas prácticas pedagógicas en los procesos de educación del cuerpo. Universidad de Antioquia (Col.), CNPq (Br.) e INDEPORTES Antioquia.

Moreno, W; Pulido, S; Gómez, J; Cardona, H; Largo, D; Díaz, N; Betancur, G; Mejía, A. \& Santa, S. (2012-2014): Juegos de la Calle, una Buena Práctica referenciable en los procesos de educación del cuerpo en Colombia. Universidad de Antioquia (CODI), CNPq (Br.) e INDEPORTES Antioquia.

Moreno, W; Pulido, S; Vásquez, A; Galvis, N; Díaz, N; Betancur, G; López, A; Hincapié, A. (2013): Juegos Recreativos Tradicionales de la Calle. Un caballo de Troya en la cultura de la ciudad. Universidad de Antioquia (CODI) e INDEPORTES Antioquia. Funámbulos Editores, Medellín.

Novelino, J. (2002): El Alma de las WebQuest.

http://www.webquest.futuro.usp.br

Olivera, B. (1989): La Educación Física y el deporte: Por una coexistencia específica. En Apunts Educació Física i Esports , Nº 16-17 junio Septiembre, 1989.

Palma, D. (1992): La sistematización como estrategia de conocimiento en la educación popular. CEAAL. Santiago de Chile, 1992

Ruiz, L.D. (2001): La sistematización de prácticas. Sistematización de la experiencia de convivencia del Liceo Nacional marco Fidel Suárez.

Torres, C \& Torres María. (2007): El Juego como estrategia de aprendizaje en el aula. Universidad de los andes. 